\title{
A relação entre o uso de betabloqueadores e a exacerbação da Doença Pulmonar Obstrutiva Crônica: uma revisão integrativa
}

\author{
The relationship between the use of beta-blockers and the exacerbation of Chronic \\ Obstructive Pulmonary Disease: a literature review
}

\section{Relación entre el uso de betabloqueantes y la exacerbación de la Enfermedad Pulmonar Obstructiva Crónica: revisión de la literatura}

Taise Gonçalves Pinheiro ${ }^{*}$, Paulo Sergio Leahy Andrade Junior ${ }^{1}$, Maria Clara Almondes de Azevedo ${ }^{1}$, Emanuelle Almeida Silva Viana ${ }^{1}$, Barbara Bernardes Magalhães ${ }^{2}$, Lucca Vieira Souza ${ }^{2}$, Brenner Marcus Alves Ferreira ${ }^{3}$, Yuri Magalhães Fernandes², Flávia Almeida dos Santos Gusmão4, Emanuel Messias Felix Neves ${ }^{5}$.

\section{RESUMO}

Objetivo: Discutir a relação entre o uso dos betabloqueadores e a exacerbação da Doença Pulmonar Obstrutiva Crônica (DPOC). Métodos: Trata-se de uma Revisão Integrativa realizada nas bases de dados Scielo, Lilacs e Pubmed. Os descritores escolhidos foram "beta blockers", "Chronic Obstructive Pulmonary Disease" e "exacerbation", juntamente com os operadores booleanos "AND". Após a análise e aplicação dos métodos de inclusão e exclusão, foram selecionados doze artigos para realização da coleta dos dados. Resultados: Dos doze artigos analisados nenhum referiu que houve um aumento da taxa de exacerbações da DPOC em pacientes que fizeram uso desses medicamentos. Sete artigos mostraram em seus resultados que os pacientes com DPOC que fizeram uso desses fármacos tiveram menos probabilidade de ter exacerbações. Além disso, alguns dos artigos selecionados mostram que os betabloqueadores seletivos apresentam melhores resultados nesses pacientes. Considerações Finais: $O$ uso dos betabloqueadores cardiosseletivos pode ser mantido em pacientes com DPOC que tenham uma indicação bem estabelecida para o seu uso, visto que não há evidência de que eles aumentem as taxas de exacerbação e hospitalização nesses pacientes.

Palavras-chave: Betabloqueadores, Doença Pulmonar Obstrutiva Crônica, Exacerbação.

\section{ABSTRACT}

Objective: Discuss the relationship between the use of beta-blockers and the exacerbation of Chronic Obstructive Pulmonary Disease (COPD). Methods: This is an Integrative Review carried out in the Scielo, Lilacs and Pubmed databases. The chosen descriptors were "beta blockers", "Chronic Obstructive Pulmonary Disease" and "exacerbation", together with the Boolean operators "AND". After analyzing and applying the inclusion and exclusion methods, twelve articles were selected for data collection. Results: Of the twelve articles analyzed, none article reported that there was an increase in the rate of exacerbations of COPD in patients who used these drugs. Seven articles showed in their results that patients with COPD who used these drugs were less likely to have exacerbations. In addition, some of the selected articles show that selective beta-blockers have better results in these patients. Final Considerations: The use of cardioselective betablockers can be maintained in patients with COPD who have a well-established indication for their use, since there is no evidence that they increase the rates of exacerbation and hospitalization in these patients.

Keywords: Beta blockers, Chronic Obstructive Pulmonary Disease, Exacerbation.

\footnotetext{
${ }^{1}$ Faculdades Santo Agostinho (FASA), Vitória da Conquista - BA. *E-mail: taisepinheiro_vc@hotmail.com

2 Universidade Estadual da Bahia (UESB), Vitória da Conquista - BA.

${ }^{3}$ Faculdade Santo Agostinho (FASAI), Itabuna - BA.

4 Universidade Federal da Bahia (UFBA), Vitória da Conquista - BA.

${ }^{5}$ Faculdades Integradas do Norte de Minas (FUNORTE), Montes Claros - MG.
} 


\section{RESUMEN}

Objetivo: Discuta la relación entre el uso de betabloqueantes y la exacerbación de la Enfermedad Pulmonar Obstructiva Crónica (EPOC). Métodos: Se trata de una Revisión Integrativa realizada en las bases de datos Scielo, Lilacs y Pubmed. Los descriptores elegidos fueron "betabloqueantes", "Enfermedad Pulmonar Obstructiva Crónica" y "exacerbación", junto con los operadores booleanos "Y". Luego de analizar y aplicar los métodos de inclusión y exclusión, se seleccionaron doce artículos para la recolección de datos. Resultados: De los doce artículos analizados, ninguno informó que hubo un aumento en la tasa de exacerbaciones de la EPOC en los pacientes que usaban estos fármacos. Siete artículos mostraron en sus resultados que los pacientes con EPOC que usaban estos fármacos tenían menos probabilidades de tener exacerbaciones. Además, algunos de los artículos seleccionados muestran que los betabloqueantes selectivos tienen mejores resultados en estos pacientes. Consideraciones finales: El uso de betabloqueantes cardioselectivos puede mantenerse en pacientes con EPOC que tienen una indicación bien establecida para su uso, ya que no hay evidencia de que aumenten las tasas de exacerbación y hospitalización en estos pacientes.

Palabras clave: Betabloqueantes, Enfermedad Pulmonar Obstructiva Crónica, Exacerbación.

\section{INTRODUÇÃO}

As doenças crônicas não transmissíveis (DCNT) representam atualmente umas das maiores causas de morbimortalidade no mundo. Dentre essas, as doenças cardiovasculares, a diabetes mellitus, neoplasias e as doenças respiratórias crônicas são responsáveis pela grande maioria dos óbitos. Da mesma forma, no Brasil, as DCNT são as principais causas de mortalidade. Essas doenças geram um grande ônus ao Sistema Único de Saúde (SUS), visto que aumentam ainda mais as filas de espera por consultas, exames especializados e cirurgias. O tratamento das DCNT consiste em mudanças de hábitos de vida, por vezes necessitando de tratamento medicamentoso e essa terapêutica pode controlar a doença, bem como reduzir seus custos e dar melhor qualidade de vida desses indivíduos (DUNCAN BB, et al., 2012).

Os betabloqueadores fazem parte do arsenal terapêutico do tratamento das doenças cardiovasculares. Eles foram descobertos em 1958 por James Black, e desde então têm sido muito utilizados no tratamento dessas doenças, além de outras patologias (OLIVER E, et al., 2019). Nos últimos anos essa classe medicamentosa tem sido amplamente estudada e seus efeitos benéficos têm sido confirmados em diversas patologias, como a insuficiência cardíaca, infarto agudo do miocárdio, hipertensão arterial e arritmia cardíaca (MARTINEZ-MILLA J, et al., 2019).

Existem 3 gerações de betabloqueadores: os não seletivos; os cardiosseletivos, que são antagonistas seletivos de $\mathrm{B} 1$; e os vasodilatadores, os quais bloqueiam os receptores $\mathrm{B} 1$ e promovem vasodilatação (OLIVER E, et al., 2019). A ação dos betabloqueadores se dá por seus efeitos antagonistas, que impedem a ligação das catecolaminas ao seu sítio de ação, tendo como consequência a inibição da estimulação dos receptores beta adrenérgicos (BOSCO FAP e BRAZ JRC, 2001). Dessa forma, essa classe medicamentosa apresenta efeitos cardioprotetivos por diminuir os efeitos prejudiciais da hiperatividade simpática. (ARGULIAN E, et al., 2019).

A Doença Pulmonar Obstrutiva Crônica (DPOC) é uma desordem respiratória de grande impacto na morbidade e mortalidade a nível global e é dividida em períodos estáveis e instáveis. Os períodos instáveis são os períodos de exacerbação da doença e se caracterizam por eventos agudos no curso natural da doença com intensificação dos sintomas de dispneia, tosse, expectoração, além de outros sintomas (MARCHIORI RC, et al., 2010).

As alterações características da DPOC são a obstrução progressiva das vias aéreas devido à inflamação presente, associada à destruição do parênquima pulmonar. Acredita-se que essa resposta inflamatória exacerbada seja secundária a inalação de partículas e gases tóxicos, principalmente a fumaça do cigarro. Além da inflamação, há evidências de que haja um desequilíbrio entre os radicais livres de oxigênio e a atividade antioxidante, resultando em uma sobrecarga oxidativa nos pulmões. Como consequência desse processo há lesão celular, hipersecreção mucosa e aumento da inflamação pulmonar (DOURADO VZ, et al., 2006). 
Considerando que os betabloqueadores não seletivos causam vasoconstrição com consequente aumento da resistência periférica e broncoconstrição por ação do efeito do medicamento nos receptores B2 dos brônquios, seu uso pode aumentar o número de hospitalizações e exacerbações de algumas doenças respiratórias, ou até mesmo diminuir a resposta a terapia de resgate com agonistas adrenérgicos beta 2 em pacientes asmáticos e dpocíticos (BAKER JG e WILCOX RG, 2016).

Dessa maneira, reconhecendo complexidade na coexistência dessas duas patologias de alta incidência e prevalência, bem como a capacidade de interveniência do tratamento inadequado na qualidade de vida dos pacientes, essa pesquisa tem como objetivo discutir e revisar a relação entre o uso de betabloqueadores e a exacerbação da DPOC.

\section{MÉTODOS}

Trata-se de uma revisão integrativa da literatura, haja vista que visa conhecer determinado assunto a partir de uma ampla análise de estudos prévios (SOUZA MT, et al., 2010). Para isso, realizou-se uma pesquisa de abordagem qualitativa nos bancos de dados Scientific Eletronic Library Online (Scielo), Literatura Latino Americana e do Caribe em Ciências da Saúde (Lilacs) e Pubmed, utilizando os seguintes descritores: "beta blockers" AND "Chronic Obstructive Pulmonary Disease" AND "exacerbation".

A busca foi efetuada entre os meses de janeiro e março de 2021. Inicialmente, não foram encontrados artigos nas bases de dados Scielo e Lilacs, e 114 artigos foram encontrados no Pubmed. Após análise dos artigos e aplicação dos critérios de elegibilidade foram selecionados 12 artigos (Figura 1). Como critérios de inclusão para essa pesquisa foram adotadas a abordagem sobre betabloqueadores em pacientes com DPOC e exacerbações respiratórias. Os critérios de exclusão foram estudos fora do período determinado, entre os anos de 2009 e 2020, e estudos com informações incompletas ou irrelevantes para a pesquisa. Também foram excluídos artigos duplicados, bem como artigos de revisão.

Figura 1 - Fluxograma descrevendo o processo de busca e seleção dos artigos.

\begin{tabular}{|l|l|l|}
\hline \multicolumn{2}{|c|}{ Estratégia de busca para as bases de dados Scielo, Pubmed e Lilacs } \\
\hline SciElo: $\mathrm{N}=0$ & PubMed: $\mathrm{N}=114$ & Lilacs: $\mathrm{N}=0$ \\
\hline \multicolumn{3}{|c|}{ Aplicação de critérios de inclusão de exclusão } \\
\hline SciElo: $\mathrm{N}=0$ & PubMed: $\mathrm{N}=12$ & Lilacs: $\mathrm{N}=0$ \\
\hline
\end{tabular}

Fonte: Pinheiro TG, et al., 2021.

\section{RESULTADOS}

Dentre doze artigos selecionados, cinco apresentam em suas conclusões que os betabloqueadores são capazes de diminuir as exacerbações da DPOC em pacientes que tem alguma doença cardíaca concomitantemente. Quatro desses artigos mostram que os betabloqueadores seletivos são menos propensos a causar essas exacerbações e um deles não encontrou diferenças na taxa de exacerbação de pacientes com DPOC sem doenças cardiovasculares que fizeram uso do metoprolol. Os demais estudos selecionados negaram ou não encontraram risco de exacerbações em pacientes dpocíticos com uso de betabloqueadores (Quadro 1). 
Quadro 1- Síntese e caracterização dos principais achados em artigos incluídos nesse estudo de revisão.

\begin{tabular}{|c|c|c|}
\hline $\mathbf{N}$ & Autor (Ano) & Evidências produzidas \\
\hline 1 & $\begin{array}{l}\text { SHORT PM, et al. } \\
(2011)\end{array}$ & $\begin{array}{c}\text { Estudo de coorte retrospectivo. Beta bloqueadores cardiosseletivos podem } \\
\text { reduzir as exacerbações dos pacientes com DPOC, além dos benefícios } \\
\text { atribuídos ao tratamento da doença cardiovascular. }\end{array}$ \\
\hline 2 & $\begin{array}{l}\text { ANGELONI E, et } \\
\text { al. (2012) }\end{array}$ & $\begin{array}{l}\text { Estudo de coorte prospectivo. A administração de betabloqueadores em } \\
\text { pacientes com DPOC submetidos a cirurgia de revascularização do miocárdio } \\
\text { não aumentou as taxas de exacerbação da DPOC. }\end{array}$ \\
\hline 3 & $\begin{array}{l}\text { FARLAND MZ, et } \\
\text { al. (2013) }\end{array}$ & $\begin{array}{l}\text { Estudo de coorte retrospectivo. Pacientes com DPOC com prescrição de } \\
\text { betabloqueadores eram menos propensos a ter uma exacerbação da doença. }\end{array}$ \\
\hline 4 & $\begin{array}{l}\text { PUENTE-MAESTU } \\
\text { L, et al. (2014) }\end{array}$ & $\begin{array}{c}\text { Estudo multicêntrico. Pacientes com DPOC com IC ou doença arterial } \\
\text { coronariana (DAC) tem menos exacerbações e visitas ao Pronto Socorro e } \\
\text { são menos sintomáticos se usarem betabloqueadores. }\end{array}$ \\
\hline 5 & $\begin{array}{l}\text { KUBOTA Y, et al. } \\
\text { (2015) }\end{array}$ & $\begin{array}{l}\text { Ensaio retrospectivo não randomizado de centro único. O uso de bisoprolol } \\
\text { reduziu a incidência de Insuficiência Cardíaca Congestiva (ICC) e/ou } \\
\text { exacerbação da DPOC em comparação com o carvedilol em paciente com } \\
\text { DPOC e IC. }\end{array}$ \\
\hline 6 & $\begin{array}{l}\text { BHATT SP, et al. } \\
\qquad(2016)\end{array}$ & $\begin{array}{l}\text { Estudo multicêntrico observacional. O uso de betabloqueadores por } \\
\text { indivíduos com DPOC está associado a uma redução das exacerbações, } \\
\text { independente da gravidade de obstrução do fluxo de ar. }\end{array}$ \\
\hline 7 & $\begin{array}{l}\text { DUFFY S, et al. } \\
\qquad(2017)\end{array}$ & $\begin{array}{l}\text { Estudo de coorte retrospectivo. O estudo em questão não encontrou } \\
\text { evidências de que os betabloqueadores aumentam as exacerbações da } \\
\text { DPOC em pacientes com doenças cardíacas ou não. }\end{array}$ \\
\hline 8 & $\begin{array}{l}\text { HUANG YL, et al. } \\
\qquad(2017)\end{array}$ & $\begin{array}{l}\text { Estudo caso controle. Betabloqueadores seletivos podem ser prescritos com } \\
\text { cautela para pacientes com DPOC e doença cardiovascular, no entanto, } \\
\text { betabloqueadores não seletivos não devem ser prescritos para pacientes com } \\
\text { DPOC. }\end{array}$ \\
\hline 9 & $\begin{array}{l}\text { BHATT SP, et al. } \\
\qquad(2017)\end{array}$ & $\begin{array}{l}\text { Estudo de coorte prospectivo. Em pacientes com DPOC moderada a muito } \\
\text { grave, a geometria cardíaca determinada por tomografia sem contraste pode } \\
\text { ser usada para prever a redução na frequência de exacerbação associada ao } \\
\text { uso de } \beta \text {-bloqueadores. }\end{array}$ \\
\hline 10 & $\begin{array}{l}\text { ZVIZDIC F, et al. } \\
\qquad(2019)\end{array}$ & $\begin{array}{l}\text { Estudo de caso controle. O uso de betabloqueadores seletivos no tratamento } \\
\text { de comorbidades cardiovasculares representa uma melhor abordagem } \\
\text { farmacológica para pacientes com DPOC no estágio GOLD } 2 \text { (moderado). }\end{array}$ \\
\hline 11 & $\begin{array}{l}\text { DRANSFIELD MT, } \\
\quad \text { et al. (2019) }\end{array}$ & $\begin{array}{l}\text { Ensaio clínico randomizado. O uso do metoprolol não influenciou na taxa de } \\
\text { exacerbação de pacientes com DPOC sem indicação estabelecida para o uso } \\
\text { de betabloqueadores. }\end{array}$ \\
\hline 12 & $\begin{array}{l}\text { RASMUSSEN DB, } \\
\text { et al. (2020) }\end{array}$ & $\begin{array}{c}\text { Estudo de coorte. O uso de betabloqueadores não foi associado ao aumento } \\
\text { do risco de exacerbação da DPOC após infarto agudo do miocárdio, } \\
\text { independente da gravidade da DPOC. }\end{array}$ \\
\hline
\end{tabular}

Fonte: Pinheiro TG, et al., 2021.

\section{DISCUSSÃO}

Os betabloqueadores têm sido usados há décadas na terapêutica de doenças cardiovasculares. $\mathrm{Na}$ terapêutica da Hipertensão Arterial Sistêmica (HAS), essa classe era utilizada como medicamentos de primeira escolha, juntamente com diuréticos. Contudo, atualmente o seu uso tem sido seletivo, não sendo mais a primeira escolha para o tratamento da HAS (BORTOLOTTO LA e COLOMBO FMC, 2009). Todavia, eles ainda têm sido uma das melhores escolhas no tratamento da insuficiência cardíaca, doença arterial coronariana, fibrilação arterial e na hipertensão complicada (DÉZSI CA e SZENTES V, 2017).

O mecanismo de ação desses medicamentos está relacionado ao efeito antagônico que ele exerce nos receptores beta-adrenérgicos em diversos órgãos. Esses receptores são acoplados à proteína $\mathrm{G}$ e são estimulados pelas catecolaminas endógenas (OLIVER E, et al., 2019). Existem 3 tipos de receptores do tipo 
beta, sendo eles os tipos RA-B1, B2 e B3. Os receptores do tipo RA-B1 são encontrados em sua grande maioria no coração, à medida que os receptores dos tipos B2 são encontrados predominantemente no músculo liso e os do tipo B3 foram encontrados em células de tecido adiposo marrom de ratos (BORTOLOTTO LA e COLOMBO FMC, 2009).

Esses receptores são estimulados por catecolaminas que são liberadas dos neurônios simpáticos pósganglionares e da medula da supra renal. Uma vez estimulados, a proteína G é ativada. Essa por sua vez, ativa a adenilciclase que converte o ATP em AMP cíclico. Posteriormente o AMP cíclico pode fosforilar o componente proteico dos canais de cálcio dependentes de voltagem, com a passagem de íons cálcio para 0 citoplasma com consequente ativação intracelular (SILVA AS e ZANESCO A, 2010).

Uma vez estimulados, uma série de cascatas são ativadas provocando alterações em diversas partes do corpo (RANG HP e DALE MM, 2016). A estimulação dos receptores B2, por exemplo, causa dilatação de vasos sanguíneos e brônquios, relaxamento do trato gastrointestinal, relaxamento uterino, relaxamento do músculo detrusor da bexiga, além de aumentar a frequência e a contratilidade cardíaca. Enquanto isso, a estimulação de receptores do tipo B1 interferem mais na força e contração cardíaca (RANG HP e DALE MM, 2016). Sendo assim após estimulo desses receptores há aumento do débito cardíaco, secundário ao aumento do volume ejetado e da frequência cardíaca (BORTOLOTTO LA e COLOMBO FMC, 2009).

Os betabloqueadores não seletivos se ligam aos receptores B1 e B2-AR, e apresenta efeito inotrópico negativo, entretanto, quando ele se liga aos receptores B2-AR causa vasoconstrição com consequente aumento da resistência periférica e broncoconstrição, o que pode afetar a função pulmonar de pacientes com asma e DPOC. (OLIVER E, et al., 2019). Posteriormente foram desenvolvidos os betabloqueadores de segunda geração, a exemplo do atenolol e metoprolol, que tem seus efeitos principalmente nos receptores B1, tendo ação mínima nos receptores B2, não provocando os efeitos adversos que ocorrem com o uso de betabloqueadores não seletivos, contudo, quando em doses elevadas, eles também podem ter ação nesses receptores (OLIVER E, et al., 2019).

Além desses, existem também os medicamentos de terceira geração, que manifestam suas ações ao antagonizar os receptores alfa-1 periféricos (BORTOLOTTO LA e COLOMBO FMC, 2009). Eles têm adicionado às suas propriedades a ação vasodilatadora e podem se ligar especificamente aos receptores $B 1$, ou ter sua ação complementada por outros mecanismos, como atividade agonista dos receptores A1-AR (OLIVER E, et al., 2019).

Os betabloqueadores podem ter diversos efeitos colaterais, principalmente os não seletivos, dentre eles o paciente pode apresentar: broncoespasmo, distúrbios de condução atrioventricular, vasoconstrição periférica, insônia, depressão psíquica, astenia e disfunção sexual. Por esse motivo o seu uso em pacientes com asma, DPOC e bloqueio atrioventricular de segundo e terceiro graus devem ser cautelosamente avaliados (MALACHIAS MVB, et al., 2016).

Existe uma incerteza sobre a prescrição de betabloqueadores quanto aos pacientes com DPOC por conta do medo de que esses medicamentos possam induzir o broncoespasmo e piorar a função pulmonar (ETMINAN M et al., 2012). Esses pacientes tem uma limitação do fluxo aéreo progressiva e não totalmente reversível que está associada a uma resposta inflamatória anormal dos pulmões. O processo inflamatório crônico causado por essa doença pode causar bronquite crônica e enfisema pulmonar, caracterizado por redução da elasticidade do pulmão e consequente alteração na sua função (SOUSA CA, et al., 2011).

As exacerbações da DPOC podem ser classificadas conforme a gravidade, sendo leves, moderadas ou graves. As agudizações leves são aquelas em que o paciente tem intensificação dos sintomas, com melhora após uso de broncodilatadores de resgate ou com a antecipação dos medicamentos em uso. Nas exacerbações moderadas o paciente também tem um aumento da necessidade de medicação, contudo, necessita de ajuda médica para resolução do quadro. Já as exacerbações graves são aquelas em que o paciente precisa ser hospitalizado (CARDOSO AP, 2013).

As causas da exacerbação em pacientes dpocíticos podem ser divididas em causas de origem respiratória e as causas de origem não respiratória. As causas respiratórias incluem as infecções respiratória, 
pneumotórax, tromboembolismo pulmonar, inalação de irritantes, ou deterioração da própria doença de base (LIMA PCS et al, 2009). Dentre as causas não respiratórias mais comuns podem ser citadas a ICC, cardiopatias isquêmicas, arritmias, infecções não respiratórias, colapso vertebral, traumatismos, refluxo gastroesofágico, desnutrição, miopatias, uso de drogas, ansiedade e uso de sedativos, sendo as doenças cardiovasculares importante causa de exacerbações (MARCHIORI RC, 2010).

As doenças cardiovasculares e a DPOC compartilham fatores genéticos e fatores de risco, como o tabagismo e a idade avançada (RABE KF, 2018). Além disso, existem outras relações entre DPOC e doenças cardiovasculares envolvendo vários fatores. Um desses fatores é a hiperinsuflação que compromete a função pulmonar. Com isso, a função cardíaca também é comprometida pelo aumento da pressão cardiopulmonar, o que pode resultar em insuficiência ventricular direita e comprometimento do enchimento do ventrículo esquerdo (RABE KF, 2018).

A hipoxemia causada pela DPOC, como consequência da alteração da relação ventilação-perfusão (V/Q), é outro fator que relaciona a DPOC e as doenças cardiovasculares, visto que a hipoxemia pode levar a vasoconstrição pulmonar e aumentar a pressão cardiopulmonar. Esse estado também leva ao remodelamento vascular, podendo evoluir para uma disfunção diastólica do ventrículo direito. Além disso aumenta a inflamação sistêmica, condição importante na fisiopatologia de doenças cardiovasculares (KENT BD et al, 2011).

Para Smith MC e Wrobel JP (2014), as comorbidades relacionadas com a DPOC tem impacto significante na qualidade de vida e na sobrevida desses pacientes. Existe um grande número de pacientes com DPOC que já tiveram um IAM, visto que esses pacientes tem uma maior probabilidade de ter essa complicação, e isso eleva as taxas de mortalidade nesses indivíduos. Pacientes com insuficiência cardíaca também tem maior risco de serem hospitalizados ou atendidos em ambulatórios especializados, do que pacientes que não tem IC, uma vez que a descompensação dessa comorbidade também se relaciona com maiores taxas de exacerbações e mortalidade (SMITH MC e WROBEL JP, 2014).

A segurança do uso desses fármacos nesses pacientes foi avaliada em alguns estudos. O resultado obtido por Duffy S, et al. (2017) foi que não foram encontradas evidências de que os betabloqueadores podem aumentar as taxas de exacerbação da DPOC em pacientes com doenças cardiovasculares, ou não (DUFFY $\mathrm{S}$, et al., 2017). Angeloni E, et al. (2012) analisaram o uso desses medicamentos em pacientes com DPOC submetidos a cirurgia de revascularização do miocárdio, e Rasmussen DB, et al. (2020) acompanhou pacientes dpocíticos com infarto agudo do miocárdio prévio em uso de betabloqueadores. Nenhum dos estudos associou o uso dos betabloqueadores a um aumento na taxa da exacerbação da DPOC nesses pacientes (ANGELONI E, et al., 2012; RASMUSSEN DB, et al., 2020).

Um outro estudo de coorte retrospectivo realizado com 412 pacientes avaliou a incidência e a gravidade das exacerbações da DPOC nesses pacientes, os quais foram divididos em dois grupos, o primeiro com 166 pacientes que faziam uso de betabloqueadores e o segundo com 246 pacientes que não faziam uso de betabloqueadores. Esse estudo mostrou que os usuários de betabloqueadores eram menos propensos a ter exacerbações da DPOC, contudo não encontrou diferença entre o número de exacerbações em pacientes fazendo uso de betabloqueadores com diferente cardiosseletividade (FARLAND MZ, et al., 2013).

Alguns dos estudos tentaram avaliar quais desses pacientes poderiam se beneficiar do uso desse medicamento. Para Bath SP, et al. (2016), os betabloqueadores estão associados a uma diminuição nas exacerbações em pacientes com DPOC independente do grau de obstrução do fluxo de ar. Por outro lado, um ensaio de caso controle realizado por Zvizdic F, et al. (2016) mostrou que entre os pacientes com DPOC estágio 3 não houve diferença no número de exacerbações entre os pacientes que usaram os betabloqueadores e os que usaram a digoxina e o verapamil entre os pacientes. Todavia, entre os pacientes com DPOC estágio 2, os que fizeram uso dos betabloqueadores seletivos tiveram menos exacerbações do que os que fizeram uso de outros fármacos.

Além desses, um estudo multicêntrico envolvendo 256 pacientes com DPOC que já tivessem indicação de betabloqueadores, mostrou que àqueles que continuaram fazendo uso desses medicamentos tiveram menos 
exacerbações, menos visitas ao pronto socorro e também eram pacientes menos sintomáticos. Apesar dos benefícios relatados com o uso dos betabloqueadores nesses pacientes esse mesmo estudo relata que ainda existe uma subprescrição dos betabloqueadores em pacientes dpocíticos que tem a indicação do seu uso (PUENTE-MAESTU L, et al., 2014). O menor número de exacerbações nesses pacientes provavelmente está relacionado ao tratamento das comorbidades cardiovasculares, mostrando que os riscos e benefícios adquiridos com o uso dos betabloqueadores devem ser pesados.

Dos estudos analisados, alguns também mostram a superioridade dos betabloqueadores cardiosseletivos em relação aos não seletivos. Em um ensaio retrospectivo, foram divididos pacientes com insuficiência cardíaca descompensada e com DPOC e classificados de acordo com o medicamento que eles estavam fazendo uso no momento da alta hospitalar: o carvedilol, que é um betabloqueador não seletivo; o bisoprolol, que é um betabloqueador altamente seletivo para os receptores do tipo B1; além do grupo dos pacientes que não fizeram uso dos betabloqueadores. Nesse estudo, a taxa de mortalidade foi maior em pacientes que não fizeram uso dos betabloqueadores. Além disso a taxa de exacerbação de ICC e da DPOC foi maior em pacientes tratados com carvedilol em comparação com o bisoprolol (KUBOTA Y, et al., 2015).

Um estudo realizado por Huang YL, et al. (2017) mostrou que os usuários de betabloqueadores cardiosseletivos tiveram um risco menor de exacerbações graves do que os que faziam uso de betabloqueadores não seletivos. Evidenciou, também, que os pacientes que fizeram uso do betaxolol, um betabloqueador seletivo, tiveram um risco significativamente menor de exacerbações graves do que os demais indivíduos. Dois betabloqueadores não seletivos foram associados ao maior risco de exacerbações, o labetalol e o propranolol. Dessa forma esse estudo concluiu que os betabloqueadores seletivos podem ser prescritos com cautela para pacientes que tenham DPOC e doença cardiovascular, no entanto o uso de betabloqueadores não seletivos não se mostrou seguro para esses pacientes.

Short PM, et al. (2011) avaliaram 5977 pacientes com mais de 50 anos, e com diagnóstico de DPOC, por um período médio de 4,35 anos. Esse ensaio mostrou redução geral da mortalidade em $22 \%$ em pacientes que faziam o uso de betabloqueadores cardiosseletivos em comparação com os pacientes que fizeram apenas o uso de terapia inalatória beta-agonista de curta ação ou antimuscarínicos de curta ação, além da redução na taxa de exacerbações. Os resultados encontrados mostraram que os pacientes foram beneficiados pela terapia com os betabloqueadores independente de terem doença cardiovascular evidente ou não.

Para Lipworth B, et al. (2016) os efeitos benéficos dos betabloqueadores nas exacerbações podem envolver outros mecanismos que vão além de suas funções cardioprotetoras, podendo estar relacionados à redução na liberação de citocinas inflamatórias sistêmicas, como inteleucina-6, além de diminuir a quimiotaxia dos neutrófilos e a produção de radicais livres de oxigênio.

Ainda não existem estudos suficientes que comprovem que o uso de betabloqueadores em pacientes com DPOC sem doenças cardiovasculares sejam beneficiados pelo uso desses medicamentos. Um estudo clínico randomizado publicado em 2019, por Dransfield MT, et al. (2019) avaliou 532 pacientes entre 40 e 85 anos com diagnóstico confirmado de DPOC e sem indicação estabelecida para uso de betabloqueadores. Ao final do estudo concluiu-se que não houve diferença entre o tempo até a primeira exacerbação da DPOC entre o grupo de pacientes que fizeram uso do metoprolol e o grupo de pacientes que fizeram uso do placebo. Além disso, a hospitalização por exacerbação foi mais comum entre os pacientes que fizeram uso do metoprolol.

Um dos estudos analisados fez uso de exames de imagem para determinar a geometria cardíaca, e a partir desse parâmetro avaliar quais pacientes com DPOC poderiam ser beneficiados com a terapia com medicamentos betabloqueadores. A relação do índice de volume do ventrículo direito pelo índice de volume do ventrículo esquerdo aparece no estudo como uma sugestão de método para distinguir pacientes que podem se beneficiar do uso dos betabloqueadores e dos que não teriam benefício significativo (BHATT SP, et al., 2017).

Muitos desses estudos mostram que os benefícios obtidos com o uso de betabloqueadores em pacientes com DPOC estão relacionados apenas ao efeito cardioprotetor desses fármacos com consequente diminuição 
da exacerbação das doenças cardiovasculares, alegando dessa forma que não haveria benefícios com o uso desses medicamentos para pacientes que não tem uma indicação estabelecida para o seu uso. Todavia, algumas pesquisas mostram resultados favoráveis no uso de betabloqueadores também em pacientes dpocíticos que não tem comorbidades cardiovasculares, sendo, assim, necessária a realização de novos estudos clínicos randomizados que orientem o profissional com relação a prescrição de betabloqueadores em pacientes com DPOC para melhorar a qualidade de vida desses indivíduos.

\section{CONSIDERAÇÕES FINAIS}

Os betabloqueadores inovaram o tratamento das comorbidades cardiovasculares. Contudo, a classe não cardiosseletiva é mais propensa a causar efeitos colaterais em pacientes com doença pulmonar obstrutiva, gerando nos profissionais de saúde receio de causar iatrogenia na prescrição aos seus pacientes. Diante disso, utilizar betabloqueadores cardiosseletivos se mostra uma alternativa benéfica aos pacientes com DPOC que possuam indicações bem estabelecidas para o uso desses medicamentos, podendo até mesmo diminuir as exacerbações dos sintomas respiratórios, bem como a mortalidade nesse grupo. Nessa perspectiva, devem ser construídos mais estudos que analisem as exacerbações em pacientes dpocíticos com doenças cardiovasculares, a fim de contribuir para o aperfeiçoamento no manejo dessas patologias coexistentes.

\section{REFERÊNCIAS}

1. ANGELONI E, et al. $\beta$-Blockers improve survival of patients with chronic obstructive pulmonary disease after coronary artery bypass grafting. Ann Thorac Surg, 2013; 95(2): 525-31.

2. ARGULIAN E, et al. Misconceptions and Facts About Beta-Blockers. The American Journal of Medicine, 2019; 132(7): 816-819.

3. BAKER JG, WILCOX RG. $\beta$-Blockers, heart disease and COPD: current controversies and uncertainties. Thorax, 2017; 72(3): 271-276.

4. BHATT SP, et al. $\beta$-Blockers are associated with a reduction in COPD exacerbations. Thorax, 2016; 71(1): 8-14.

5. BHATT SP, et al. Cardiac Morphometry on Computed Tomography and Exacerbation Reduction with $\beta$-Blocker Therapy in Chronic Obstructive Pulmonary Disease. Am J Respir Crit Care Med, 2017; 196(11): 1484-1488.

6. BORTOLOTTO LA, COLOMBO FMC. Betabloqueadores adrenérgicos. Rev Bras Hipertens, 2009; 16(4): $215-220$.

7. BOSCO FAP, BRAZ JRC. Beta-Bloqueadores em Anestesiologia: Aspectos Farmacológicos e Clínicos. Revista Brasileira de Anestesiologia, 2001; 51(5): 431-447.

8. CARDOSO AP. Exacerbação da DPOC. Pulmão RJ, 2013; 22(2): 60-64.

9. DÉZSI CA, SZENTES V. The Real Role of $\beta$-Blockers in Daily Cardiovascular Therapy. American Journal of Cardiovascular Drugs, 2017; 17(5): 361-373.

10. DOURADO VZ, et al. Manifestações sistêmicas na doença pulmonar obstrutiva crônica. J Bras Pneumol, 2006; 32(2): 161-71.

11. DRANSFIELD MT, et al. Metoprolol for the Prevention of Acute Exacerbations of COPD. N Engl J Med, 2019; 381(24): 2304-2314.

12. DUFFY S, et al. Effect of beta-blockers on exacerbation rate and lung function in chronic obstructive pulmonary disease (COPD). Respir Res, 2017; 18(1): 124.

13. DUNCAN BB, et al. Doenças crônicas não transmissíveis no Brasil: prioridade para enfrentamento e investigação. Rev. Saúde Pública [online], 2012; 46(1): 126-134.

14. ETMINAN M, et al. Beta-blocker use and COPD mortality: a systematic review and meta-analysis. BMC Pulm Med, 2012; 4(12): 48.

15. FARLAND MZ, et al. $\beta$-Blocker use and incidence of chronic obstructive pulmonary disease exacerbations. Ann Pharmacother, 2013; 47(5): 651-6.

16. HUANG YL, et al. Impact of selective and nonselective beta-blockers on the risk of severe exacerbations in patients with COPD. Int J Chron Obstruct Pulmon Dis, 2017; 12: 2987-2996.

17. KENT BD, et al. Hypoxemia in patients with COPD: cause, effects, and disease progression. International Journal of COPD, 2011; 6: 199-208.

18. KUBOTA Y, et al. Impact of $\beta$-blocker selectivity on long-term outcomes in congestive heart failure patients with chronic obstructive pulmonary disease. Int J Chron Obstruct Pulmon Dis, 2015; 10: 515-23.

19. LIMA PSC, et al. Perfil de patógenos nas exacerbações agudas da doença pulmonar obstrutiva crônica: resultados preliminares. Pulmão RJ, 2009; 18(3): 127-132.

20. LIPWORTH B, et al. Beta-blockers in COPD: time for reappraisal. Eur Respir J, 2016; 48(3): 880-8.

21. MALACHIAS MVB, et al. 7th Brazilian Guideline of Arterial Hypertension. Arq Bras Cardiol., 2016; 107(3): 1-83.

22. MARCHIORI RC, et al. Diagnóstico e tratamento da DPOC exacerbada na emergência. Revista Científica AMRIGS, 2010; 54(2): 214-223. 
23. MARTÍNEZ-MILLA J, et al. Role of Beta-blockers in Cardiovascular Disease in 2019. Revista Española de Cardiologia, 2019; 72(10): 844-852.

24. OLIVER E, et al. Beta-blockers: Historical Perspective and Mechanisms of Action. Revista Española de Cardiologia, 2019; 72(10): 853-862.

25. PUENTE-MAESTU L, et al. Multicentric study on the beta-blocker use and relation with exacerbations in COPD. Respir Med, $2014 ;$ 108(5): 737-44.

26. RABE KF, et al. Cardiovascular disease and COPD: dangerous liaisons?. European Respiratory Review, 2018; 27(149): 180057.

27. RANG HP, DALE MM. Rang e Dale: Farmacologia. 8nd ed. Elsevier Editora, 2016; 1939.

28. RASMUSSEN DB, et al. Beta-blocker use and acute exacerbations of COPD following myocardial infarction: a Danish nationwide cohort study. Thorax, 2020; 75(11): 928-933.

29. SHORT PM, et al. Effect of beta blockers in treatment of chronic obstructive pulmonary disease: a retrospective cohort study. BMJ, 2011; 342: d2549.

30. SILVA AS, ZANESCO A. Exercício físico, receptores $\beta$-adrenérgicos e resposta vascular. J. vasc. bras. [online], 2010; 9(2): 47-56.

31. SMITH MC, WROBEL JP. Epidemiology and clinical impact of major comorbidities in patients with COPD. Int J Chron Obstruct Pulmon Dis, 2014; 9: 871-88.

32. SOUSA CA et al. Doença pulmonar obstrutiva crônica e fatores associados em São Paulo, SP, 2008-2009. Rev. Saúde Pública [online], 2011; 45(5): 887-896.

33. SOUZA MT, et al. Revisão integrativa: o que é e como fazer. Einstein, 2010; 8(1): 102-6.

34. ZVIZDIC F, et al. Beta-blocker Use in Moderate and Severe Chronic Obstructive Pulmonary Disease. Med Arch, 2019; 73(2): 72-75. 